\title{
Performance of SNAPPE-II score in neonatal sepsis: an experience from a tertiary care center
}

\author{
Moumita Samanta ${ }^{1}$, Chiranjib Biswas ${ }^{2}$, Nishith Kumar Pal' ${ }^{3}$, Soma Sarkar ${ }^{4}$, \\ Avranil Goswami ${ }^{4}$, Avijit Hazra ${ }^{5}$, Arijit Bhowmik², Tapas Kumar Sabui ${ }^{6}$ \\ Departments of ${ }^{1}$ Pediatrics and ${ }^{3}$ Microbiology, Nil Ratan Sircar Medical College \& Hospital (NRSMCEH) Medical College, Kolkata; \\ Departments of ${ }^{2}$ Pediatrics and ${ }^{4}$ Microbiology, Medical College and Hospital, Kolkata; ${ }^{5}$ Department of Pharmacology, Institute of \\ Postgraduate Medical Education and Research, Kolkata; ${ }^{6}$ Department of Pediatrics, RGKAR Medical College and Hospital, Kolkata, \\ India.
}

\begin{abstract}
Background and objectives. The Score for Neonatal Acute Physiology II with Perinatal Extension (SNAPPE-II) is a vital tool for prognostication in newborns. The study was conducted with the hypothesis that the performance of the SNAPPE-II score might be affected by the presence of sepsis in newborns admitted with possible early onset septicemia and whether score performance varies between culture positive and culture negative sepsis.
\end{abstract}

Methods. The prospective observational study was conducted over a period of 1 year (January 2014 to January 2015) in neonates presenting with clinical suspicion of sepsis to the Sick Newborn Care Unit (SNCU) of a tertiary care hospital in Eastern India.

Results. SNAPPE-II score cut-off of $\geq 20$ offered the highest sensitivity of $74.5 \%$ with specificity $48.3 \%$, PPV $27.6 \%$ and NPV $87.7 \%$. Comparison of mortality proportions between the two subgroups defined by this cut-off returned $\mathrm{p}=0.005$ with OR 3.47 (95\% 1.40 to 8.64). No significant association was found between SNAPPE-II score and blood culture results; mean scores for culture positive $(25.16 \pm 15.6)$ and negative groups $(24.49 \pm 15.6)$ were comparable $(\mathrm{p}=0.920)$.

Conclusions. At a cut-off value of $\geq 20$ in presence of sepsis, SNAPPE-II score offers acceptable indices to predict mortality outcome. Prediction of outcome by SNAPPE-II score is not affected by positive or negative blood culture sepsis.

Key words: neonatal sepsis, SNAPPE score, blood culture.

Neonatal sepsis continues to be a major cause of neonatal morbidity and mortality worldwide. ${ }^{1-4}$ Globally, neonatal sepsis is responsible for 4 million deaths annually and $99 \%$ of them occur in developing countries. ${ }^{5}$ Very often, the signs of infections may be absent or subtle or hard to detect and fatal septicemia may occur with little warning. ${ }^{6}$ Hence timely diagnosis of neonatal sepsis is critical.

Over the years blood culture has been considered the gold standard for diagnosis

\footnotetext{
Tapas Kumar Sabui
}

tsabui@gmail.com

Received 25th February 2019, accepted 8th April 2019. of neonatal septicemia. However, it is time consuming and to circumvent this drawback various prognostication scores or tools have been developed based on risk factors, acute physiological changes and biochemical parameters. These strategies help predict onset of acute sickness in newborns and help monitoring while on therapy. Management begins at the earliest suspicion of sepsis and newborns are subjected to level II or level III care based on the severity of illness scores. The Score for Neonatal Acute Physiology (SNAP) II with Perinatal Extension (SNAPPE-II) by Richardson et al. ${ }^{7,8}$ and modified over the years, comprises of physiology-based indicators which are measured within the first 12 hours of admission. ${ }^{9}$ 
In our study we explored the association between SNAPPE-II score and mortality in neonatal sepsis of our referral hospital setting. Neonates with sepsis usually have more physiological instability which may affect the scores. To test this hypothesis we compared scores of newborns with sepsis (clinical or culture proven) to those without sepsis. Previous studies have shown significantly higher mortality rates in blood culture positive than culture negative neonates. ${ }^{10}$ Thus, we further decided to test the score performance in blood culture positive versus negative neonates. This could have implications in clinical practice in assessment of score and its predictability in the presence of sepsis.

Henceforth to explore these possibilities, we conducted this study with the hypothesis that the performance of the SNAPPE-II score might be affected by the presence of sepsis in newborns admitted with possible early onset septicemia and whether score performance varies between culture positive and culture negative sepsis.

\section{Material and Methods}

A prospective observational study was conducted over a period of 1 year (January 2014 to January 2015) in neonates presenting with clinical suspicion of sepsis to the Sick Newborn Care Unit (SNCU) of a tertiary care hospital in Eastern India. The study was approved by the Institutional Ethics Committee for clinical research on 2 January 2014 (MC/Kol/IEC/227/12014).

Cases were recruited on alternate days of the week, excluding the weekends, through purposive sampling. Parental written informed consent was obtained after selection and before enrolment of subjects. The inclusion criteria were neonates with features suggestive of neonatal sepsis like respiratory distress (excluding hyaline membrane disease), labored breathing, cyanosis, apnea in a previously healthy baby, temperature instability, lethargy, refusal to suck, abdominal distension, feed intolerance, subtle seizure / vacant stare and suspected necrotizing enterocolitis (NEC). Appropriate investigations were done. Neonates with congenital infection, congenital metabolic disease, prior antibiotic use, and unavailable blood culture or sepsis screen reports were excluded.

The severity of illness of each infant was systematically assessed for the first 12-hour period after enrolment. The time of enrolment once the neonate was diagnosed to have sepsis was considered as ' 0 ' hour. The worst value for each scoring parameter in the 12hour period was taken for determining the total SNAPPE-II score. Data on the perinatal extension parameters were collected from perinatal assessment records. The SNAPPEII score was thereafter calculated by the resident doctor trained in neonatology and subsequently verified by a senior investigator. Other variables recorded were sex of the baby, place and type of delivery, postnatal age at admission and intrauterine growth restriction. This information were collected from labor theatre records in case of inborn and discharge summary in case of outborn babies. The subjects were closely monitored every day till discharge or death. A structured case report form was used for capturing data.

Sepsis screen (which includes total leukocyte count, absolute neutrophil count, immature/ total neutrophil, micro-ESR and C-reactive protein) ${ }^{1}$ and blood culture (using BacT/ ALERT 3D 60 automated blood culture system) were performed for all neonates recruited. Neonates with two or more sepsis screen parameters positive were considered to have screen positive sepsis. Positive blood cultures were further analyzed for species identification and antimicrobial susceptibility testing using VITEK $^{\circledR} 2$ instrument (of Compact Biomerieux, Inc. Durham, North Carolina, USA). In neonates with clinical features suggestive of meningitis, or culture proven sepsis, cerebrospinal fluid (CSF) examination was performed. The cohort for analysis was subdivided into blood culture positive (definitive sepsis) and blood culture negative (includes clinical sepsis where sepsis 
screen is negative and probable sepsis where screen is positive) groups.

Statistical analysis: Data were analyzed with SPSS statistics software version 17 (Illinois, Chicago: SPSS Inc., 2008). Numerical variables were compared between subgroups by Student's independent samples t-test while Fisher's exact test was employed for comparison of independent proportions. For these comparisons, $\mathrm{p}$ value less than 0.05 was considered to be statistically significant. Ninety five percent confidence interval $(95 \% \mathrm{CI})$ values and Odds ratio (OR) have been presented where deemed relevant. Sensitivity, specificity, positive predictive value (PPV), negative predictive value (NPV), likelihood ratio positive (LR+) and likelihood ratio negative (LR-) were calculated to evaluate performance of different cut-offs of the SNAPPE-II score in predicting mortality. A receiver operating characteristics (ROC) curve analysis was also done to discriminate the best performing cut-off of SNAPPE-II score to predict outcome.

\section{Results}

Of the 246 neonates enrolled, 21 were excluded (2 had glucose-6-phosphate dehydrogenase deficiency, 1 galactosemia, 5 congenital infections, and rest 13 received antibiotics before enrolment) leaving an analysis cohort of 225 neonates, of whom 124 (55.1\%) were males. The mean gestational maturity of the whole cohort was $35.1 \pm 2.5$ weeks (range $28-40$ weeks), with 96 (42.7\%) neonates being term ( $\geq 37$ weeks gestational maturity). The characteristics of whole group are shown in Table I.

Aerobic blood culture was done for each enrolled neonate at the onset of suspicion of sepsis. Blood culture results $(n=225)$ showed growth of organism in 97 neonates. However, 8 growths (6 being coagulase negative Staphylococcus, 1 Micrococcus and 1 Candida) were considered as contaminants in the background of non-corroborative clinical features and favorable outcome in all without antibiotics. Hence, the total number of neonates with growth of pathogenic organism in blood culture (culture positive) was 89 (39.6\%); while the rest 136 neonates $(60.4 \%)$ were culture negative. Blood culture growth was dominated by gram negative organism in $75(84.3 \%)$ and gram positive in the rest. Klebsiella species was by far the most common organism identified (54\%); followed by Pseudomonas (13.5\%), E. coli $(10.1 \%)$ and Acinetobacter (6.7\%). Staphylococcus aureus $(13.79 \%)$ and Enterococcus (2.2\%) were the gram positive organisms isolated.

Respiratory distress was the most common presenting symptom (69.8\%); with $43.9 \%$ of these being culture positive $(p=0.041$ chi-square; OR 1.9, 95\% CI 1.02-3.46). Feed intolerance seen in $5.3 \%$ neonates was also significantly more in the culture positive group than in the culture negative group $(66.7 \%$ vs. $33.3 \%$ respectively; $\mathrm{p}=0.048$ Pearson chi-square; OR 3.3, 95\% CI 0.95-11.17) Table II. The mean gestational maturity of newborns who survived were modestly but significantly different from that for the expired neonates $(35.4 \pm 2.3$ weeks vs. $34.1 \pm 2.8$ weeks respectively; $\mathrm{p}=0.002 ; 95 \%$ CI of the difference 0.48 to 2.07). Mean birth weight in survivors was also significantly more than for expired neonates $(2276.3 \pm 730.1 \mathrm{~g}$ vs. $1808.5 \pm 659.6$ g respectively, $\mathrm{p}<0.001 ; 95 \% \mathrm{CI}$ of difference 236.4 to $699.3 \mathrm{~g}$ ). SGA neonates were significantly more prone to death than their appropriate for gestational age counterparts ( $32.4 \%$ vs. $15.6 \%$ respectively; $\mathrm{p}=0.004$; OR 2.60 ; $95 \%$ CI 1.34 to 5.03). However, survival rates in blood culture positive and negative groups were not statistically significant $(74.16 \%$ vs. $82.35 \%$ respectively; $\mathrm{p}=0.139$ ). The mean age in hours of life at SNAPPE-II measurement was $31.10 \pm 24.15$ (median 27 hours, 95\% CI 27.9334.27).

Total mortality observed in our study was 47/225 (20.9\%; 95\% CI 15.6 to $26.2 \%$ ). Comparison of SNAPPE-II scores revealed higher SNAPPE-II score in neonates who expired (range $5-73$, mean $30.4 \pm 15.7$, median 28) than in those who survived (range $0-65$, mean $23.7 \pm 15.3$, median 22.0) and this was significantly different 
Table I. Characteristics of infants according to sepsis groups (N=225).

\begin{tabular}{|c|c|c|c|}
\hline \multirow[b]{3}{*}{ Characteristics } & \multicolumn{2}{|c|}{ Sepsis groups } & \multirow[b]{3}{*}{$P$ value } \\
\hline & True sepsis & Suspected sepsis & \\
\hline & (Culture proven, $\mathrm{n}=89$ ) & (Culture negative, $\mathrm{n}=136$ ) & \\
\hline Male, n (\%) & $48(53.9)$ & $76(55.9)$ & 0.775 \\
\hline \multicolumn{4}{|l|}{ Gestational age, $\mathrm{n}(\%)$} \\
\hline$\geq 37$ weeks & $29(32.6)$ & $66(48.5)$ & 0.55 \\
\hline 32 to 36 weeks & $53(59.5)$ & $61(44.9)$ & \\
\hline$<32$ weeks & $7(7.9)$ & $9(6.6)$ & \\
\hline \multicolumn{4}{|l|}{ Birth weight, n (\%) } \\
\hline$\geq 2.5 \mathrm{~kg}$ & $33(37.1)$ & $53(38.9)$ & 0.689 \\
\hline 1.5 to $<2.5 \mathrm{~kg}$ & $41(46.1)$ & $56(41.2)$ & \\
\hline$<1.5 \mathrm{~kg}$ & 15 (16.8) & $27(19.8)$ & \\
\hline \multicolumn{4}{|l|}{ Birth weight, n (\%) } \\
\hline$>999 \mathrm{~g}$ & $89(100)$ & 135 (99.3) & 0.713 \\
\hline $750-999 \mathrm{~g}$ & 0 & $1(0.7)$ & \\
\hline$<750 \mathrm{~g}$ & 0 & 0 & \\
\hline Small for gestational age, $\mathrm{n}(\%)$ & $17(19.1)$ & $26(19.1)$ & 0.014 \\
\hline \multicolumn{4}{|l|}{ APGAR score at 5 minutes, $\mathrm{n}(\%)$} \\
\hline$\geq 7$ & $58(65.2)$ & 74 (54.4) & 0.002 \\
\hline$<7$ & $31(34.8)$ & $62(45.6)$ & \\
\hline \multicolumn{4}{|l|}{ Lowest mean arterial pressure, $\mathrm{n}(\%)$} \\
\hline$>29 \mathrm{mmHg}$ & $83(93.3)$ & $134(98.5)$ & 0.923 \\
\hline $20-29 \mathrm{mmHg}$ & $5(5.6)$ & $2(1.5)$ & \\
\hline$<20 \mathrm{mmHg}$ & $1(1.1)$ & 0 & \\
\hline \multicolumn{4}{|l|}{ Lowest temperature, $\mathrm{n}(\%)$} \\
\hline$>35.6^{\circ} \mathrm{C}$ & $39(43.8)$ & $61(44.9)$ & 0.601 \\
\hline $35-35.6^{\circ} \mathrm{C}$ & $21(23.6)$ & $33(24.2)$ & \\
\hline$<35^{\circ} \mathrm{C}$ & $29(32.6)$ & $42(30.9)$ & \\
\hline \multicolumn{4}{|l|}{$\mathrm{PaO}_{2} / \mathrm{FiO}_{2^{\prime}} \mathrm{n}(\%)$} \\
\hline$>2.49$ & $34(38.2)$ & $64(47.1)$ & 0.576 \\
\hline $1.0-2.49$ & $36(40.4)$ & $49(36.0)$ & \\
\hline $0.3-0.99$ & $19(21.2)$ & $23(16.9)$ & \\
\hline$<0.3$ & - & - & \\
\hline \multicolumn{4}{|l|}{ Lowest pH, n (\%) } \\
\hline$>7.19$ & 83 (93.6) & 132 (97.1) & 0.984 \\
\hline $7.10-7.19$ & $6(6.7)$ & $4(2.9)$ & \\
\hline$<7.10$ & 0 & 0 & \\
\hline Seizure, n (\%) & $42(47.2)$ & $51(37.5)$ & 0.025 \\
\hline \multicolumn{4}{|l|}{ Urine output, n (\%) } \\
\hline$>0.9 \mathrm{ml} / \mathrm{kg} / \mathrm{h}$ & $69(77.5)$ & $104(76.5)$ & 0.920 \\
\hline $0.1-0.9 \mathrm{ml} / \mathrm{kg} / \mathrm{h}$ & $20(22.5)$ & $32(23.5)$ & \\
\hline$<0.1 \mathrm{ml} / \mathrm{kg} / \mathrm{h}$ & 0 & 0 & \\
\hline SNAPPE II Score & $25.16 \pm 15.6$ & $24.49 \pm 15.6$ & 0.936 \\
\hline
\end{tabular}


Table II. Blood culture results according to clinical features ( $\mathrm{N}=225)$.

\begin{tabular}{lccccc}
\hline \multirow{2}{*}{ Clinical features } & \multirow{2}{*}{ Total $^{*}$} & \multicolumn{2}{c}{ Blood Culture } & P value & $\begin{array}{c}\text { Odd's ratio } \\
(95 \% \text { CI })\end{array}$ \\
\cline { 3 - 5 } & & Culture positive** & Culture negative** & & $1.88(1.02-3.46)$ \\
Respiratory distress & $157(69.8)$ & $69(43.9)$ & $88(56.1)$ & 0.041 & - \\
Lethargy, refusal to feed & $71(31.6)$ & $28(39.4)$ & $43(60.6)$ & 0.980 & - \\
Apnea & $23(10.2)$ & $11(47.8)$ & $12(52.2)$ & 0.392 & $3.3(0.95-11.17)$ \\
Feed intolerance & $12(5.3)$ & $8(66.7)$ & $4(33.3)$ & 0.048 & - \\
Abdominal distension & $11(4.9)$ & $4(36.4)$ & $7(63.6)$ & 0.824 & - \\
Suspected NEC & $6(2.7)$ & $3(50.0)$ & $3(50.0)$ & 0.596 & - \\
Subtle seizure & $4(1.8)$ & $2(50.0)$ & $2(50.0)$ & 0.666 & - \\
Temp. instability & $3(1.3)$ & $1(33.3)$ & $2(66.7)$ & 0.824 & \\
\hline
\end{tabular}

*: results are presented as $\mathrm{n}(\%)$; column percentile

${ }^{* *}$ : results are presented as $\mathrm{n}(\%)$; row percentile

NEC: necrotizing enterocolitis

( $\mathrm{p}=0.008 ; 95 \% \mathrm{CI}$ of difference 1.76 to 11.69 ). In general there was progressive rise in mortality with increasing SNAPPE-II score. This trend was statistically significant $(\mathrm{p}=0.004)$ by chisquare for trend analysis. Table III indicates the performance of various SNAPPE-II cut-offs in predicting mortality in terms of standard diagnostic indices. The cut-off of $\geq 20$ offered the highest sensitivity of $74.5 \%$ with specificity 48.3\%, PPV $27.6 \%$ and NPV 87.7\%. Comparison of mortality proportions between the two subgroups defined by this cut-off returned an OR of 3.47 (95\% CI 1.40 to 8.64; $\mathrm{p}=0.005)$.

No significant association was found between SNAPPE-II score and blood culture results ( $25.16 \pm 15.6$ in culture positive vs. $24.49 \pm$ 15.6 in culture negative group; $\mathrm{p}=0.920$ ). Performance of SNAPPE-II score with cut-off $\geq 20$ was further assessed in different gestational age subgroups and it was found that score performed best in 32-36 weeks gestational age group in predicting the outcome irrespective of blood culture results. SNAPPE-II scores compared between survivors and fatal cases in various gestational maturity categories, showed a statistically significant difference for the 32-36 week gestational age band in both blood culture positive and blood culture negative cases (Table IV) but not for other bands. When ROC curve analysis was done for different subgroups to assess discriminatory power of SNAPPE-II score to predict mortality outcome, the area under curve (AUC) was reasonable only for 32-36 weeks gestational maturity group (AUC 0.705) (Fig. 1).

\section{Discussion}

Results of the whole cohort revealed significant association between mortality in neonatal sepsis and increasing SNAPPE-II score $(\mathrm{p}=0.004)$. Similar results have been reported by other authors. ${ }^{11-14}$ RA et al. ${ }^{11}$ reported that SNAPPE II has the best performance in predicting first

Table III. Performance of SNAPPE-II score in predicting mortality at various cut-offs.

\begin{tabular}{lcccccc}
\hline SNAPPE-II score cut-offs & $\geq 20$ & $\geq 30$ & $\geq 35$ & $\geq 40$ & $\geq 50$ & $\geq 60$ \\
\hline Sensitivity & $74.5 \%$ & $42.5 \%$ & $31.9 \%$ & $25.5 \%$ & $12.8 \%$ & $4.2 \%$ \\
Specificity & $48.3 \%$ & $64 \%$ & $74.7 \%$ & $87 \%$ & $95.5 \%$ & $97.7 \%$ \\
Positive predictive value & $27.6 \%$ & $23.8 \%$ & $25 \%$ & $34.3 \%$ & $42.8 \%$ & $33.3 \%$ \\
Negative predictive value & $87.7 \%$ & $80.8 \%$ & $81 \%$ & $81.6 \%$ & $80.6 \%$ & $79.4 \%$ \\
Likelihood ratio positive & 1.44 & 1.2 & 1.26 & 1.98 & 2.84 & 1.89 \\
Likelihood ratio negative & 0.53 & 0.9 & 0.91 & 0.85 & 0.91 & 0.98 \\
\hline
\end{tabular}


Table IV. SNAPPE II score in gestational maturity categories stratified by blood culture results.

\begin{tabular}{lccc}
\hline Gestational age & Outcome & SNAPPE-II score & P value \\
\hline Blood culture positive sepsis $(\mathrm{n}=89)$ & & & \\
\hline$<32$ weeks $(\mathrm{n}=18)$ & Survived $(\mathrm{n}=7)$ & $31.0 \pm 21.42$ & 0.762 \\
& Died $(\mathrm{n}=11)$ & $34.0 \pm 19.29$ & \\
32 to 36 weeks $(\mathrm{n}=42)$ & Survived $(\mathrm{n}=32)$ & $27.2 \pm 18.86$ & 0.025 \\
& Died $(\mathrm{n}=10)$ & $43.1 \pm 18.84$ & \\
$\geq 37$ weeks $(\mathrm{n}=29)$ & Survived $(\mathrm{n}=27)$ & $30.9 \pm 17.57$ & 0.699 \\
& Died $(\mathrm{n}=2)$ & $36.0 \pm 21.21$ & \\
\hline Blood culture negative sepsis $(\mathrm{n}=136)$ & & & 0.241 \\
\hline$<32$ weeks $(\mathrm{n}=22)$ & Survived $(\mathrm{n}=17)$ & $32.8 \pm 18.12$ & \\
& Died $(\mathrm{n}=5)$ & $43.6 \pm 14.98$ & 0.026 \\
32 to 36 weeks $(\mathrm{n}=47)$ & Survived $(\mathrm{n}=38)$ & $29.4 \pm 17.69$ & \\
& Died $(\mathrm{n}=9)$ & $44.2 \pm 16.37$ & 0.650 \\
$\geq 37$ weeks $(\mathrm{n}=67)$ & Survived $(\mathrm{n}=57)$ & $27.5 \pm 17.97$ & \\
& Died $(\mathrm{n}=10)$ & $30.3 \pm 16.94$ &
\end{tabular}

$\mathrm{P}$ value in the last column is for comparison between survivors and fatal cases.

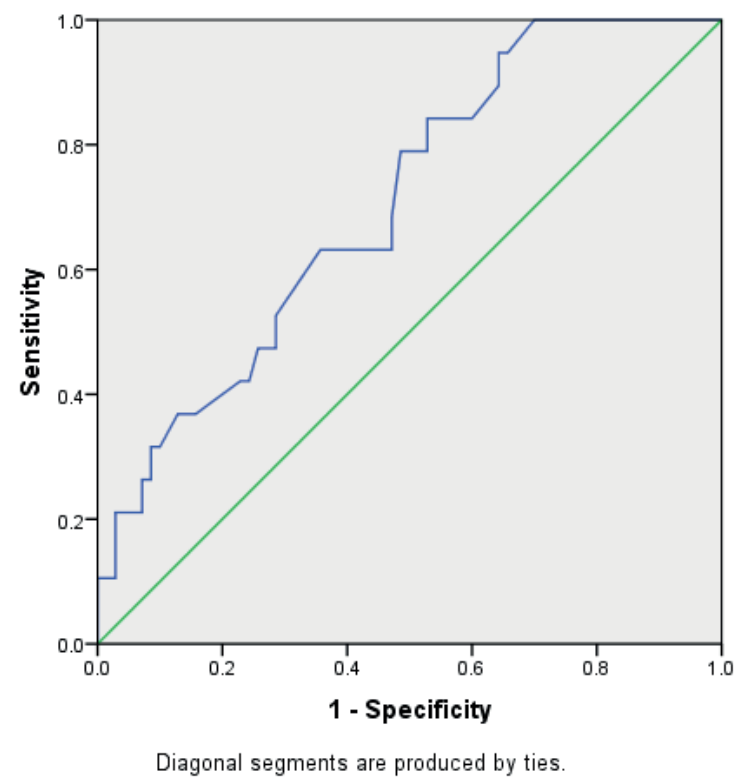

Fig. 1. Receiver operating curve for SNAPPE-II Score to predict mortality in neonates at gestational age of 32 to 36 weeks.

6-days mortality. However, the cut-off point of SNAPPE II scoring system to predict mortality has been found to differ in various studies. While RA et al. ${ }^{11}$ suggested a cut-off point 30 we found a cut-off point of 20 for maximum sensitivity of mortality prediction with fairly acceptable specificity. Jain et al. ${ }^{15}$ observed that, as the score increased to 40 and above probabilities of mortality increased and it was maximum with score of 80 and above.

Ramirez et al. ${ }^{13}$ found that SNAPPE-II score showed higher values for newborns who died compared to survivors, with analysis of the ROC curve showing an area under the curve for SNAPPE II of 0.77 (CI 95\% 0.69-0.86).

On analysis of SNAPPE-II score among the demographic subgroups of the entire cohort, we found that performance of SNAPPE-II score in predicting mortality was statistically significant only in 32-36 weeks gestational maturity group. SNAPPE-II did not seem to predict outcome well in those with gestational age $<32$ weeks or $>36$ weeks. Possibly, prematurity and birth asphyxia are the confounding factors that have overshadowing effect on the outcome in this regard. This could also be an incidental finding and needs further studies to prove its reproducibility.

Positivity of blood culture did not seem to alter the performance of SNAPPE-II score. In other words SNAPPE-II score predicted outcome independent of blood culture results. Moreover no significant difference of mean score in the 
two groups was found $(p=0.920)$. This is in contrary to the hypothesis that newborns with true sepsis might have greater SNAPPE score. The mortality rate in our study was $20.1 \%$ which is less than that reported in various studies from India, (37\% to $47 \%)$ among the early onset sepsis cases. ${ }^{16,17}$ This could possibly be due to a secular trend or due to changes in obstetric and neonatal care practices. No significant difference in death between blood culture positive $(25.8 \%)$ and blood culture negative (17.6\%) neonates were found in our study. This finding is unusual as previous studies have reported significantly higher mortality rates in culture positive than culture negative neonates. ${ }^{18}$ Vinay et al. ${ }^{19}$ also had a similar finding of overwhelmingly higher death rates in culture positive $(83.6 \%)$ than in culture negative $(16.4 \%)$ cases.

One of the most common causes of neonatal mortality in our country is septicemia and delayed referral to higher centers. Currently established country level guidelines for early referral include mostly the serious complications like recurrent seizures, shock, hypoglycemia etc. ${ }^{20}$ Our study analysis shows that SNAPPEII scores does perform well in predicting outcome but are not useful in differentiating true sepsis from suspected (or clinical sepsis). The existing country level guidelines for early referral are still good enough.

Like any other study our study also has its share of limitations. Firstly sample size was smaller and skewed resulting in difficult subgroup analyses. Late onset sepsis was not included to avoid various confounding variables involved in transport of these newborns. Healthy newborns had they been included as matched control group would have helped to compare SNAPPEII score between the septic (both suspected and true) and non-septic healthy groups.

In conclusion we can say that, notwithstanding the limitations, at a cut-off value of $\geq 20$ in presence of sepsis, SNAPPE-II score offers acceptable indices to predict mortality outcome. Prediction of outcome by SNAPPE-II score is not affected by presence or absence of true sepsis. SNAPPE II score of $\geq 20$ could be a possible cutoff for early referral of sick newborns to higher centers, particularly between 32-36 weeks of gestational age.

\section{Acknowledgement}

We thank Prasenjit Saha, Lab Technician, Deepanwita Pal, Lab Techinician and Arunava Bhattacharyya from Medical College and Hospital, Kolkata for their logistic help. We would like to thank National Health Mission for funding this project.

\section{REFERENCES}

1. Puopolo KM. Bacterial and fungal infections. In: Cloherty JP, Eichenwald EC, Stark AR (eds). Manual of Neonatal Care (6th ed). Philadelphia: Lippincott Williams \& Wilkins, 2008: 274-300.

2. Sharma M, Yadav A, Yadav S, Goel N, Chaudhary U. Microbial profile of septicemia in children. Indian J Pract Doct 2008; 5: 5-8.

3. Stoll BJ, Hansen N, Fanaroff AA, et al. Lateonset sepsis in very low birth weight neonates: the experience of the NICHD Neonatal Research Network. Pediatrics 2002; 110(2 Pt 1): 285-291.

4. Bang AT, Bang RA, Baitule SB, Reddy $\mathrm{MH}$, Deshmukh MD. Effect of home-based neonatal care and management of sepsis on neonatal mortality: field trial in rural India. Lancet 1999; 354: 1955-1961.

5. Darmstadt GL, Saha SK, Choi Y, et al; and the Bangladesh Projahnmo-2 (Mirzapur) Study Group. Population-based incidence and etiology of community-acquired neonatal bacteremia in Mirzapur, Bangladesh: an observational study. J Infect Dis 2009; 200: 906-915.

6. Shirazi H, Riaz S, Tahir R. Role of the hematological profile in early diagnosis of neonatal sepsis. Ann Pak Inst Med Sci 2010; 6: 152-156.

7. Richardson DK, Gray JE, McCormick MC, Workman K, Goldman DA. Score for neonatal acute physiology: a physiology severity index for neonatal intensive care. Pediatrics 1993; 91: 617-623.

8. Richardson DK, Corcoran JD, Escobar GJ,Lee M SK. SNAP-II and SNAPPE-II: Simplified newborn illness severity and mortality risk scores. J Pediatr 2001; 138: $92-100$. 
9. Harsha SS, Archana BR. SNAPPE- II (Score for Neonatal Acute Physiology with Perinatal Extension-II) in predicting mortality and morbidity in NICU. J Clin Diagn Res 2015; 9: SC10-SC12.

10. Gebrehiwot A, Lakew W, Moges F, et al. Predictors of positive blood culture and death among neonates with suspected neonatal sepsis in Gondar University Hospital, Northwest Ethiopia. Euro J Exp Bio 2012; 2: 2212-2218.

11. RA M, Etika R, Harianto A, Indarso F, Damanik $\mathrm{SM}$. The use of score for neonatal acute physiology perinatal extention II (SNAPPE II) in predicting neonatal outcome in neonatal intensive care unit. Paediatr Indones 2005; 45: 241-245.

12. Thimoty J, Hilmanto D, Yuniati T. Score for Neonatal Acute Physiology Perinatal Extension II (SNAPPE II) as the predictor of neonatal mortality hospitalized in neonatal intensive care unit. Paediatr Indones 2009; 49: 155-159.

13. Ramirez MNM, Godoy LE, Barrientos EA. SNAP II and SNAPPE II as Predictors of Neonatal Mortality in a Pediatric Intensive Care Unit: Does Postnatal Age Play a Role? Int J Pediatr 2014; 2014: 298198.
14. Reid S, Bajuk B, Lui K, Sullivan EA; NSW and ACT Neonatal Intensive Care Units Audit Group, PSN. Comparing CRIB-II and SNAPPE-II as mortality predictors for very preterm infants. J Paediatr Child Health 2015; 51: 524-528.

15. Jain S, Bansal A. SNAPPE II score for predicting mortality in a level II neonatal intensive care unit. Dicle Med J 2009; 36: 333-335.

16. Anwer SK, Mustafa S. Rapid identification of neonatal sepsis. J Pak Med Assoc 2000; 50: 94-98.

17. Chacko B, Sohi I. Early onset neonatal sepsis. Indian J Pediatr 2005; 72: 23-26.

18. Sriram R. Correlation of blood culture results with the sepsis score and the sepsis screen in the diagnosis of neonatal septicemia. Int J Biol Med Res 2011; 2: 360-368.

19. Vinay BS, Girish GN, Adhikari S, Hugara S. Evaluation of septic screen as a diagnostic tool for neonatal sepsis in a tertiary hospital at Mysore. Sch J App Med Sci 2015; 3(2G): 1005-1010.

20. Facility Based Newborn Care Operational Guide. Guidelines for Planning and Implementation. Ministry of Health and Family Welfare, Government of India, 2011. 